\title{
Core-Collapse Supernova neutrino detection prospects with the KM3NeT neutrino telescopes.
}

\author{
Marta Colomer Molla ${ }^{1,2, a}$ and Massimiliano Lincetto ${ }^{3, b}$ \\ ${ }^{1} A P C$, Univ Paris Diderot, CNRS/IN2P3, CEA/Irfu, Obs de Paris, Sorbonne Paris Cité, France \\ ${ }^{2}$ IFIC_Instituto de Física Corpuscular (CSIC_Universitat de València), Paterna, Valencia, Spain \\ ${ }^{3}$ Aix Marseille Univ, CNRS/IN2P3, CPPM, Marseille, France
}

\begin{abstract}
Core Collapse Supernovae (CCSN) are explosive phenomena that may occur at the end of the life of massive stars, releasing over $99 \%$ of the energy through neutrino emission with energies on the $10 \mathrm{MeV}$ scale. While the explosion mechanism is not fully understood, neutrinos are believed to play an important role. The only detection as of today are the 24 neutrinos from supernova SN1987A. The observation of the next Galactic CCSN will lead to important breakthroughs across the fields of astrophysics, nuclear and particle physics.

For a Galactic CCSN, the KM3NeT ORCA and ARCA detectors in the Mediterranean Sea will observe a significant number of neutrinos via the detection of Cherenkov light, mostly induced by Inverse Beta Decay (IBD) interactions in sea water. The detection of coincident photons by the 31 photomultipliers of the KM3NeT digital optical modules (DOMs) allows to separate the signal from the optical background sources.

The KM3NeT detection sensitivity to a Galactic CCSN and the potential to resolve the neutrino light-curve have been estimated exploiting detailed Monte-Carlo simulations. Specific criteria are proposed for the online triggering and the participation in the SNEWS network.
\end{abstract}

\section{Introduction}

The KM3NeT experiment consists of two Cherenkov detectors under construction in the Mediterranean Sea, ORCA and ARCA, composed of Digital Optical Modules (DOMs) each housing 31 3-inch photomultipliers (PMTs) [1]. Groups of 18 DOMs are arranged into flexible vertical lines and an array of 115 lines constitutes a building block. ORCA, off the French coast, will consist of one block with $9 \mathrm{~m}$ vertical separation between DOMs and ARCA of two blocks with $36 \mathrm{~m}$ vertical spacing between DOMs, offshore Sicily. Together, they constitute an instrumented volume at the $\mathrm{km}^{3}$ scale [2].

Although KM3NeT detectors are mainly designed for high-energy neutrino detection, the $10 \mathrm{MeV}$ scale neutrino signal from a CCSN can be identified as a simultaneous increase of the counting rate in the photomultipliers of the detector. The main interaction modes of these MeV neutrinos in water are Inverse Beta Decay, Elastic Scattering (ES) and interactions of neutrinos with oxygen atoms.

\footnotetext{
ae-mail: mcolomer@apc.in2p3.fr

be-mail: lincetto@cppm.in2p3.fr
} 
The outgoing electron/positron produced in these interactions induces Cherenkov light that can be detected by the PMTs.

\section{Flux models and simulations}

A state-of-the-art 3D simulation of the accretion phase of a $27 \mathrm{M} \odot$ and an $11 \mathrm{M} \odot$ CCSN provided by the Garching group have been considered this work [3, 4].

The time dependent CCSN neutrino spectrum is a quasi-thermal distribution, which depends on the average neutrino energy $\tilde{E}_{v}$, the neutrino luminosity $L(t)_{S N}^{v}$ and the spectral pinching shape parameter, $\alpha$ :

$$
\begin{gathered}
\frac{d \Phi^{v}}{d E_{v}}\left(E_{v}, t\right)=\frac{L(t)_{S N}^{v}}{4 \pi d^{2}} \times f\left(E_{v}, \tilde{E}_{v}(t), \alpha(t)\right), \\
f\left(E_{v}, \tilde{E}_{v}(t), \alpha(t)\right)=\frac{E_{v}^{\alpha}}{\Gamma(\alpha+1)}\left(\frac{\alpha+1}{\tilde{E}_{v}}\right)^{(\alpha+1)} \exp \left[-\frac{E_{v}(\alpha+1)}{\tilde{E}_{v}}\right],
\end{gathered}
$$

The simulation output has been used to compute the CCSN neutrino interaction rate by multiplying the flux with the latest cross section estimates in $[5,6]$ as follows:

$$
\frac{d R_{i n t, \kappa}}{d E}=N_{\kappa} \cdot \sigma_{\kappa}(E) \cdot \frac{d \Phi}{d E} \quad\left[\mathrm{~s}^{-1} \mathrm{MeV}^{-1}\right] \quad \kappa \in\left\{\mathrm{p}, \mathrm{e}^{-16},{ }^{16} \mathrm{O}\right\}
$$

A full MC simulation of CCSN neutrinos in KM3NeT has been produced and used to estimate the expected detection rate in the detector for a CCSN at an arbitrary distance, considering background rates as observed on the first ORCA and ARCA detection lines in the sea.

${ }^{40} \mathrm{~K}$ decays in sea water are one of the main background sources and they can be distinguished from a CCSN signal using coincidences between the different PMTs of a given DOM. The number of PMTs in a DOM detecting a photon within a time window of $10 \mathrm{~ns}$ is called multiplicity $(\mathrm{M})$ and is used to reject the ${ }^{40} \mathrm{~K}$ background, dominating at low multiplicities. Dedicated simulations have shown that selecting events in the multiplicity range $6 \leq \mathrm{M} \leq 10$ optimizes the signal-to-noise ratio.
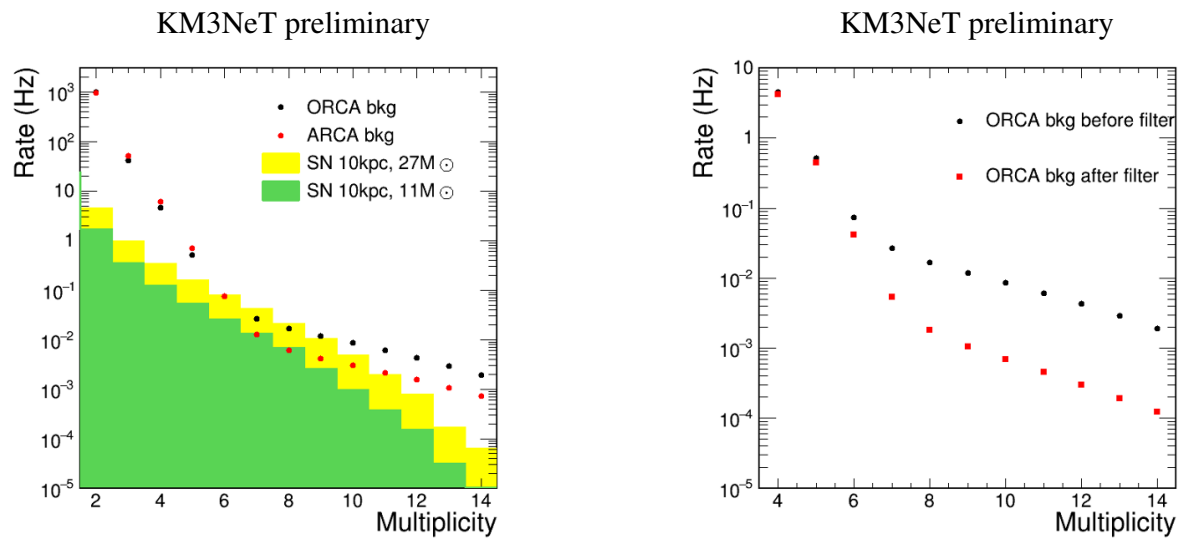

Figure 1. On the left, DOM background rates as a function of the multiplicity for ORCA (black squares) and ARCA (blue squares) from data and expectations for CCSN signal at $10 \mathrm{kpc}$ for the $27 \mathrm{M} \odot$ (yellow filled) and the $11 \mathrm{M} \odot$ (green filled) progenitors. On the right, ORCA rates before (black dots) and after the filter (red squares). 
Atmospheric muons are another prominent background producing Cherenkov tracks over long distances with high multiplicity events on multiple DOMs. Suppression of $\mathrm{M} \geq 4$ correlated coincidences between two or more DOMs on a few $\mu$ s time scale has been proven to be an effective strategy to reject the muon background. A muon filter has been developed and optimized for ORCA.

On Fig. 1 (left), it can be seen the multiplicity rates per KM3NeT DOM for signal simulations and background for both KM3NeT detectors, ARCA and ORCA. The effect of the muon filter on the ORCA background rates above $\mathrm{M}=4$ is shown on the right plot.

\section{Significance results}

The number of signal and background events when applying the multiplicity cut and the muon filtering are used to compute the detection significance as a function of the distance to the source.

KM3NeT preliminary

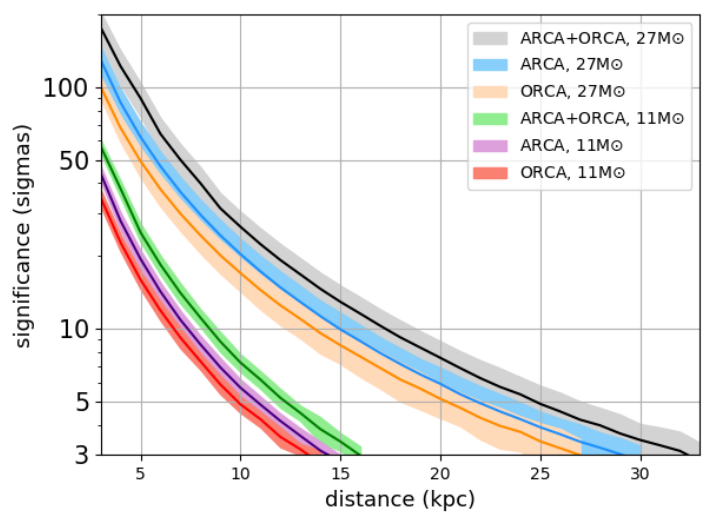

Figure 2. KM3NeT significance to a CCSN neutrino signal as a function of the distance to the source.

The results for ORCA, ARCA and the combination of both detectors are shown in Fig. 2 for the two stellar progenitors considered in this study. The more relevant systematics, coming mostly from the uncertainties on the models, and the statistical uncertainties have been taken into account. A combined significance of $5 \sigma$ is achieved at $25 \mathrm{kpc}$ for the $27 \mathrm{M} \odot$ progenitor, ensuring the coverage of the full Galaxy. In the case of the $11 \mathrm{M} \odot$ progenitor, a significance of $5 \sigma$ is reached beyond the Galactic Centre with the full ORCA detector.

\section{Trigger performance}

An approach for the real-time triggering of a CCSN neutrino burst, applicable to both ORCA and ARCA, has been evaluated and will be operational from the first phases of the detector deployment.

Data from the deployed ARCA and ORCA lines have been extensively analyzed by sampling the background on a $\tau=100 \mathrm{~ms}$ time scale in order to identify patterns in the DOM response that could mimic the CCSN signal. A robust trigger is implemented by defining the trigger level as the number of DOMs that detect a multiple coincidence in the multiplicity cut over a larger sliding time window of $n$ times $\tau$, with $n$ an integer allowing to cover the signal duration. 
Given $\rho$ the average background rate in the multiplicity cut (after muon filtering), the false rate for a given trigger level $X$ can be estimated and related to the distance to the source in the following way:

$$
\begin{gathered}
R_{B}(X)=\tau^{-1} \cdot P\left(N_{\text {lines }} \cdot n \rho \tau, X\right) \\
X=N_{\text {lines }} \cdot X_{10 k p c}(n \tau) \cdot\left(\frac{D}{10 k p c}\right)^{2}
\end{gathered}
$$

Estimated false trigger rates are shown for the ORCA detector in Fig. 3 as a function of the CCSN distance, where we highlight the $\leq 1 /$ week fake trigger requirement to participate in the global SNEWS alert network [7], for the two progenitors studied. A CCSN of $27 \mathrm{M} \odot$ could be triggered with full ORCA up to $18.6 \mathrm{kpc}$ and above the Galactic Centre for the $11 \mathrm{M} \odot \mathrm{CCSN}$.
KM3NeT preliminary

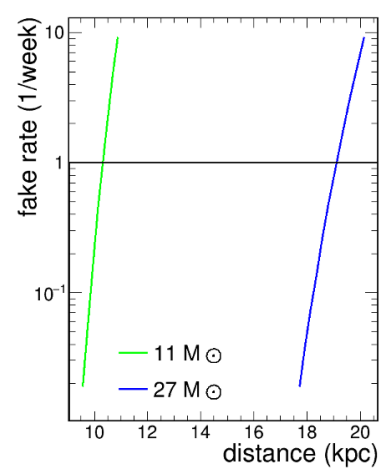

Figure 3. Fake trigger rate in ORCA as a function of the distance to the CCSN for the two progenitors studied. The black line indicates the SNEWS threshold.

\section{Sensitivity to fast time variations on the neutrino light-curve.}

Latest 3D simulations predict anisotropic hydrodynamical instabilities during the CCSN accretion phase, which may play an important role in the efficient neutrino heating [8] [9]. We focus here on the Standing Accretion Shock Instability (SASI) that could explain the observed energetic explosion. It leaves as a footprint fast time variations in the neutrino light-curve around $200 \mathrm{~ms}$ after the core bounce, with a characteristic oscillation frequency [4].

KM3NeT preliminary

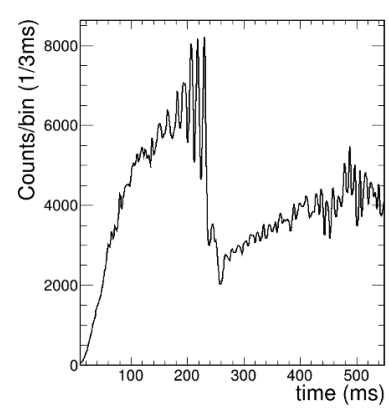

KM3NeT preliminary

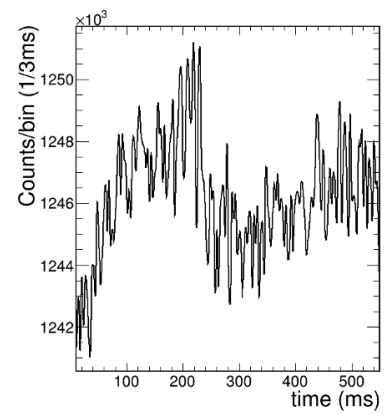

KM3NeT preliminary

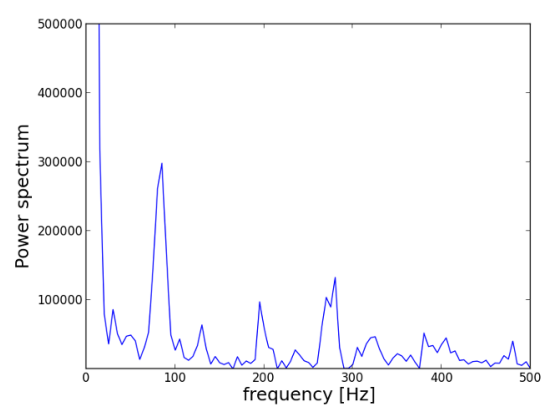

Figure 4. On the left, the expected light-curve for CCSN at 5kpc, where SASI oscillations are seen at around 200 ms. On the middle, the expected light-curve in one KM3NeT block when adding background. On the right, the power spectrum for background+signal at $5 \mathrm{kpc}$, where the peak is recovered at the SASI oscillation frequency.

The detected CCSN neutrino light-curve has been simulated for a the $27 \mathrm{M} \odot$ progenitor at 5 kpc, including all hits. Background has been included assuming a Poisson distribution, which is a preliminary an quite optimistic assumption. As in [10], a Fourier analysis has been used to recover the SASI frequency and the significance to the SASI peak has been estimated through MC pseudoexperiments. Results are given in Fig. 4. A $3 \sigma$ sensitivity to the SASI oscillation peak is obtained at 5 $\mathrm{kpc}$ for the $27 \mathrm{M} \odot$ progenitor model with one KM3NeT detection block. A more detailed description of the background will follow in a more advance analysis. 


\section{Conclusions}

The response of the KM3NeT neutrino detectors to core-collapse supernova neutrinos has been estimated by means of a complete Monte Carlo simulation and an exhaustive study of the background from the first KM3NeT datasets.

The KM3NeT combined sensitivity for a future CCSN detection has been estimated to be of $5 \sigma$ at $25 \mathrm{kpc}$ (coverage of the full Galaxy) for a $27 \mathrm{M} \odot$ stellar progenitor. Assuming an $11 \mathrm{M} \odot$ stellar progenitor, a significance of $5 \sigma$ is reached above the Galactic Centre with the full ORCA detector.

As for the online triggering capabilities, evaluated for the ORCA detector, the maximum triggering distance meeting the SNEWS false alert rate requirement has been estimated to be well beyond the Galactic Centre for the two progenitors studied.

\section{Acknowledgements}

This project is partly supported by the LabEx UnivEarthS exploratory project E9: Low energy astrophysics with KM3NeT.

\section{References}

[1] KM3NeT Collaboration, "The prototype detection unit of the KM3NeT detector", (arXiv:1510.01561) (2015)

[2] KM3NeT Collaboration, "Letter of intent for ARCA and ORCA", (arXiv:1601.07459v2) (2016)

[3] I. Tamborra et al., "Neutrino emission characteristics and detection opportunities based on threedimensional supernova simulations", Phys. Rev. D 90, 045032 (arXiv e-prints 1406.0006) (2014)

[4] I. Tamborra et al., "Neutrino signature of supernova hydrodynamical instabilities in three dimensions", Phys. Rev. Lett. 111,121104 (arXiv e-prints 1307.7936) (2013)

[5] A. Strumia and F. Vissani, Phys. Lett. B 564 (2003) 42

[6] G. Radel and R. Beyer, Mod. Phys. Lett. A, 08 (1993) 12

[7] K.Scholberg, "The Supernova Early Warning System", (arXiv:0803.0531) (2008)

[8] A. Marek, H.-T. Janka and E. Müller, "Equation-of-state dependent features in shock-oscillation modulated neutrino and gravitational-wave signals from supernovae", Astron. Astrophys. 496, 66 (2009)

[9] H.T.Janka, "Neutrino-driven Explosions", arXiv:1702.08825 (2017)

[10] J.Miganda, "Detecting Fast Time Variations in the Supernova Neutrino Flux with HyperKamiokande", arXiv:1609.04286 (2015) 